\title{
SLOW FLARES AND ERUPTIVE PHENOMENA IN EARLY STAGES
} OF STELLAR EVOLUTION

\author{
M.A. IBRAHIMOV, V.S.SHEVCHENKO \\ Astronomical Institute \\ Uzbek Academy of Sciences \\ USSR
}

\begin{abstract}
Correlations between different eruptive fhenomena ( fast and slow flares, large-scale eruptive variations up to fuor phenomenon) in stellar instability during stage of evolution are analysed $A$ conclusion is drawn on the difference in natures of eruptive phenomena lasting some ten minutes and slower variations lasting more than one day.
\end{abstract}

Analysis of the sample of about 40000 photoelectric observations of the $T$ Tauri stars and related objects, obtained in 1978-88 at the Mount Majdanak, revealed the absence of eruptive phenomena (including the brightness drops or "anti-flares") on a time scale of 10 to 100 days. This fact initiated us to analyse the time distributions of the faster flares and a slower brightness variations.

Five common type of the eruptive pheromena at the early stages of stellar evolution can be characterized by amplitude, duration and morphology.

1) The rapid flares of the UV Ceti-type stars in the solar vicinity and of the flare stars in stellar clusters and associations. The total number of these stars is ower 1400 [1]

2) The slow flares of the flare stars mentioned above. 50 slow flares of 38 flare stars were mentioned in the publications before 1986 [1-6]. 11 "relatively slow" flares with the rising time of about $15-20$ minutes were reported by Hojaev [7] for Tau T3-association and by Tsvetkova [8] for $\gamma$ Cyg region.

3) The flares of $T$ Tau-type stars, which originally were reported by Haro and Chavira [9] for VY Ori in 1953. 17 flares of $13 \mathrm{~T}$ Tau stars were detected by Hojaev [10] in Tau T3- assotiation during the 750 hours of observations. The evidences of the flares were reported by Zajtseva [11] for DF Tau, Shevchenko [12] for T Tau, Furtig and Wenzel [13] for RW Aur. Inspite of the finding by Hojaev [10] and 
Parsamian [14], one may conclude that the flares of $T$ Tau stars are quite rare events: about one flare over 100 hours of observations [11,12]. Our analysis of the Majdanak data sample suggest that this estimate may be too optimistic. The "flares" of DF Tau with the duration of 3 to 5 days may be caused by modulation of light of the rotating star by a hot spot $[15,16]$. Apparently, the periodical "flares" of other objects, e.g.TZ Ori [5], may be of the same origin. It seems that all the available data do not provided the information about the flares of $T$ Tau stars longer than 1 day.

Table 1. Classification of the eruptive phenomena according to the specific properties of the process

\begin{tabular}{cccc}
\hline $\begin{array}{c}\text { Type of events } \\
\text { (examples) }\end{array}$ & $\begin{array}{c}\text { Number } \\
\text { of stars time (day) }\end{array}$ & $\begin{array}{c}\text { Rverall } \\
\text { duration } \\
\text { (day) }\end{array}$ & $\begin{array}{c}\text { Amplit. } \\
\text { (das }\end{array}$
\end{tabular}

1. Rapid flares (UV

Cet type and flare stars in aggrega$1400 \quad 10^{-5}<t<10^{-2} \quad 10^{-4}-10^{-2} \leq 4.5$ tes).

2. Slow flares

(the same ob-

40

$10^{-2}<t<10^{-1} \quad 0.1-0.5 \leq 2$

jects).

3. T Tau star

flares (T,DF Tau

RW Aur, VY Ori)

$\sim 40 \quad 10^{-2}<t<1 ? \quad<0.5-5 \leq 0.5-1$

\section{?}

$-$

$-$

$10-100$

4. Subfuor errup-

tions (Shanal and

Sugano objects.

$\sim 100-150$

$\sim 10^{3} \quad \leq 3-3.5$

EX Lup, $Z \mathrm{CMa}$,

DR, RY, UZ, VY Tau)

5. Fuor eruptions

(FU Ori, V1057, V1515.

V1735 Cyg, V350 Cep

4) The fuor-like flares of the "subfuors" [17]. The light curves of these objects resemble the small scale fuor eruption with the decay time of about 2.5 yrs. The premaximum luminosity of the objects differs of that of fuors and their spectrum at maximum light is also differs of that of fuors.

5) The large scale eruptive phenomena of the FU Ori type stars (fuors). Three objects of this type is known up today 
and 2 or 3 objects are suspected to be the fuors.

In the Table 1 we have collected and classified all the eruptive phenomena according to the numbers of events and the specific properties of the process (rising time, duration and amplitude).

The absence of the eruptive phenomena with the duration of 10 to 100 days cannot be explained by any observational selection. The mean property of Majdanak data sample show that the flarelike eruptive phenomena with the duration of 10 to 100 days can more easily be observed. Therefore it is difficult to explain the existing of such rift.

\section{REFERENCES}

1. Mirzoyan,L.V. and Ohanian,G.B.(1986) 'Flare stars in stellar clusters and associations ', in L.V.Mirzoyan (ed.), Flare stars and Related Objects. Armenian Academy of Sciences, Yerevan, pp.68-78.

2. Parsamian, E.S. (1980) 'Slow flares in aggregates. I', Astrofizika 16, 87-96.

3. Parsamian, E.S. (1980) 'Slow flares in aggregates. II', Astrofizika 16, 231-241.

4. Mirzoyan,L.V. (1981) Instability and stellar evolution, V.A.Ambartsumian (ed.), Armenian Academy of Sciences, Yerevan.

5. Parsamian, E.S. and H.A.Pogosian(1986) 'Peculiar stars in the Orion association region', in L.V.Mirzoyan (ed.). Flare stars and Related Objects. Armenian Academy of Sciences, Yerevan, pp. 130-134.

6. Shevchenko V.S.(1986) 'Slow flares on flare dwarfs in the solar neighbourhud ', in L.V.Mirzoyan (ed.), Flare stars and Related Objects. Armenian Academy of Sciences, Yerevan, pp.135-137.

7. Hojaev,A.S. (1986) 'Flare stars in the TDC', in L.V.Mirzoyan (ed.), Flare stars and Related objects. Armenian Academy of Sciences, Yerevan, pp. 91-100.

8. Tsvetkova, K. (1986) 'Flare stars in the region near $\gamma$ Cygni', in L.V.Mirzoyan (ed.), Flare stars and Related Objects, Armenian Academy of Sciences, Yerevan, pp.84-90.

9. Haro, G. and Chavira, E. (1965) Vistas in Astronomy 7, 89.

10. Hojaev, A.S. (1987) ' Flares of Orion population variables in the association Taurus T3'. Astrofizika, 27, 207-217.

11. Zajtseva,G.V. (1980) 'Flares of the T Tauri-type stars', in L.V.Mirzoyan (ed.), Flare Stars,Fuors and Herbig-Haro Objects, Armenian Academy of Sciences, Yerevan, pp. 61-68. 
12. Shevchenko V.S. (1980) 'A comparative analysis of T Tau and red dwarf flares'. in L.V.Mirzoyan (ed.). Flare Stars. Fuors and Herbig-Haro Objects. Armenian Academy of Sciences , Yerevan, pp.69-75.

13. Furtig, W. and Wenzel, W. (1963) Mitt. Veranderl.Sterne 2,11 .

14. Parsamian, E.S. (1982) Philosophy Doct. Thesis, Yerevan.

15. Bertout, C.,Basri,G. and Bouvier, J. (1988) Astrophys.J. 330,350 .

16. Shevchenko V.S.(1986) 'Quasi-periodic activity $T$ Tau, the Herbig Ae/Be stars and fuors', in L.V.Mirzoyan (ed.). Flare stars and Related Objects, Armenian Academy of Sciences, Yerevan, pp.230-244.

17. Parsamian, E.S. and Gasparian L.G.(1987) 'On fuorlike variations of the Orion association stars', Astrofizika, $27,447-458$. 